\title{
A Saddlepoint Approximation to the Distribution Function of the Anderson-Darling Test Statistic
}

\author{
David E. A. Giles
}

Department of Economics, University of Victoria, B.C., Canada

April, 2000

\begin{abstract}
The Anderson-Darling goodness-of-fit test has a highly skewed and non-standard limit distribution. Various attempts have been made to tabulate the associated critical points, using both theoretical approximations and simulation methods. We show that a standard saddlepoint approximation performs well in both tails of the distribution. It is markedly superior to other theoretical approximations in the lower tail of the distribution.
\end{abstract}

Author Contact: $\quad$ Professor David Giles, Department of Economics, University of Victoria, P.O. Box 1700 STN CSC, Victoria, B.C., Canada, V8W 2Y2

e-mail: dgiles@uvic.ca; FAX: (250) 721 6214; Phone: (250) 7218540 


\section{INTRODUCTION}

The Anderson-Darling $(1952,1954)$ test for goodness-of-fit is widely used, and has good power properties. This non-parametric test is used to test the hypothesis that a sample of size $n$ has been drawn from a population with specified continuous cumulative distribution function. The distribution of the test statistic is complicated, even asymptotically, but it has been studied by a number of authors. Anderson and Darling (1954, p. 766) calculate the asymptotic critical points for significance levels of $1 \%, 5 \%$ and $10 \%$. Lewis (1961) uses an empirical approximation to the finite-sample distribution, based on Monte Carlo simulation, and provides extensive tables of critical points. He also uses Hermite-Gauss numerical integration to evaluate the theoretical asymptotic critical points, extending the result of Anderson and Darling (1954). Sinclair and Spurr (1988) derive the first four cumulants of the asymptotic distribution for the Anderson-Darling statistic, and provide a theoretical approximation to this distribution based on Zolotarev's (1961) results on limiting distributions of quadratic forms. Their approximation works well in the upper tail of the distribution, but is quite poor in the lower tail. In particular it yields areas in excess of unity over quite a range of critical values. Sinclair and Spurr deal with the latter problem by using, instead, an empirical approximation based on fitting a generalized logistic distribution to Lewis's critical values.

In this note we derive an alternative theoretical approximation to the asymptotic distribution function of the Anderson-Darling statistic. Our objective is to obtain a single theoretical result that performs well in both tails of the distribution. This approximation uses a saddlepoint formula (e.g., Daniels, 1954) of the type developed by Lugannani and Rice (1980). Saddlepoint approximations have been used with great success by many authors, and excellent recent discussions of their application to a range of distributional problems are given by Reid (1988), Goutis and Casella (1999), and Huzurbazar (1999).

In the next section we present the test statistic and some of its basic properties. Section 3 discusses the saddlepoint theory that we use here, and our principal theoretical results. Numerical evaluations follow in section 4 , together with some comparisons with other related results. Concluding comments appear in section 5 . 


\section{THE ANDERSON-DARLING STATISTIC}

For a sample of size $\mathrm{n}$, the Anderson-Darling test statistic is:

$$
A_{n}^{2}=n \int_{-\infty}^{\infty}\left[F_{n}(x)-F(x)\right]^{2} /[F(x)\{1-F(x)\}] d F(x)
$$

where $F_{n}(x)$ is the empirical distribution function, and $F(x)$ is the theoretical distribution function. The test statistic can be computed by ordering the observed $\mathrm{x}_{\mathrm{i}}$ 's so that $\mathrm{x}_{1} \leq \mathrm{x}_{2} \leq \ldots \ldots \leq \mathrm{x}_{\mathrm{n}}$, and constructing

$$
A_{n}^{2}=-n-(1 / n) \sum_{j=1}^{n}(2 j-1)\left[\ln \left(u_{j}\right)+\ln \left(1-u_{n-j+1}\right)\right]
$$

where $u_{i}=F\left(x_{i}\right) ; i=1,2, \ldots \ldots \ldots, n$.

The asymptotic test statistic is

$$
A^{2}=\underset{n \rightarrow \infty}{\operatorname{Limit}}\left(A_{n}^{2}\right)
$$

and we are concerned here with an accurate evaluation of $\operatorname{Pr} .\left(A^{2} \geq y\right)$. The upper tail of the distribution of $\mathrm{A}^{2}$ is of special interest as it relates to the rejection region for the test.

We will use the result that the characteristic function of $\mathrm{A}^{2}$ is shown by Anderson and Darling (1952,p.204)) to be:

$$
\theta(t)=[(-2 \pi i t) / \cos (0.5 \pi \sqrt{1+8 i t})]^{1 / 2}
$$

This can also be written (Anderson and Darling, 1952, p.204) as:

$$
\begin{aligned}
\theta(t) & =\prod_{j=1}^{\infty}[1-2 i t /(j(j+1))]^{-1 / 2} \\
& =\prod_{j=1}^{\infty}\left[1-2 \lambda_{j} i t\right]^{-1 / 2} ; \quad \lambda_{j}=1 /(j(j+1))
\end{aligned}
$$


which is just the characteristic function of an infinite sum of independent $\chi^{2}(1)$ variates, with weights $\lambda_{j}$. This feature of the test statistic suggests that a saddlepoint approximation to its distribution may perform well. As is noted by Wood et al. (1993, p. 680), the Lugannini-Rice saddlepoint formula is known “....to be a remarkably good approximation to the cumulative distribution function (CDF) of a sum of independent random variables."

From (1), the cumulant generating function for $\mathrm{A}^{2}$ is:

$$
\kappa(t)=\log [\theta(t)]=-0.5 \sum_{j=1}^{\infty} \log \left[1-2 \lambda_{j} t\right]
$$

and from (2) Sinclair and Spurr (1988, p.1190) show that the associated skewness coefficient is 5.5865. Lewis (1961, p.1118) also notes that for practical purposes, $A^{2} \in[0,8]$.

\section{A SADDLEPOINT APPROXIMATION}

To obtain a saddlepoint approximation we need the first two derivatives of the cumulant generating function which, from (2), are:

$$
\begin{aligned}
& \kappa^{\prime}(t)=\sum_{j=1}^{\infty}\left[\lambda_{j} /\left(1-2 \lambda_{j} t\right)\right] \\
& \kappa^{\prime \prime}(t)=2 \sum_{j=1}^{\infty}\left[\lambda_{j} /\left(1-2 \lambda_{j} t\right)\right]^{2}
\end{aligned}
$$

Then, to determine the saddlepoint approximation to $\operatorname{Pr} .\left(A^{2} \geq y\right)$, we solve the saddlepoint equation, $\kappa^{\prime}(t)=y$, and use the unique solution $(t=\hat{t})$ to evaluate:

$$
\hat{w}=\{2[\hat{t} y-\kappa(\hat{t})]\}^{1 / 2} \operatorname{sgn} .(\hat{t})
$$

and

$$
\hat{u}=\hat{t}\left\{\kappa^{\prime \prime}(\hat{t})\right\}^{1 / 2}
$$


where sgn. $(x)= \pm 1,0$ if $x$ is positive, negative or zero.

Then, the saddlepoint approximation to the c.d.f. of $\mathrm{A}^{2}$ is:

$$
\operatorname{Pr} .\left(A^{2} \geq y\right) \approx 1-\Phi(\hat{w})+\phi(\hat{w})\left[\hat{u}^{-1}-\hat{w}^{-1}\right] \quad,
$$

where $\phi(\cdot)$ is the standard Normal density function, and $\Phi(\cdot)$ is the corresponding c.d.f.. So, the first step is to solve, for each y-value of interest, the saddlepoint equation:

$$
S_{y}(t)=\sum_{j=1}^{\infty}\left[\lambda_{j} /\left(1-2 \lambda_{j} t\right)\right]-y=0 \quad ; \quad \lambda_{j}=1 /(j(j+1))
$$

The solution, $\hat{t}$, is well-defined, because

$$
\operatorname{Limit}_{j \rightarrow \infty}\left[\lambda_{j} /\left(1-2 \lambda_{j} t\right)\right]=0
$$

and it is unique, because

$$
S_{y}^{\prime}(t)=\kappa^{\prime \prime}(t)=2 \sum_{j=1}^{\infty}\left[\lambda_{j} /\left(1-2 \lambda_{j} t\right)\right]^{2}>0 .
$$

Equation (8) is readily solved numerically by the Newton-Raphson algorithm, for example, using (9). Alternatively, a line-search may be used (e.g., Wood et al., 1993, p.683).

\section{NUMERICAL EVALUATIONS}

The numerical results that follow were generated with double-precision FORTRAN code written by the author. The code incorporates routines from Press et al. (1992) to evaluate $\Phi(\cdot)$ using the "error function". The code was compiled and optimized with the Lahey (1992) F77L compiler and executed on a P.C. with an Intel-PentiumIII/450 processor. 
Table 1 compares our saddlepoint approximation for $\operatorname{Pr} .\left(A^{2} \geq y\right)$ with the theoretical approximation of Sinclair and Spurr (1988, p. 1191). The critical values are taken from Lewis (1961). As can be seen, our results are marginally superior to those of Sinclair and Spurr in the right tail of the distribution, but dramatically superior in the left tail.

Table 1. Theoretical Approximations to Tail Areas

\begin{tabular}{llcl}
\hline Critical Value & $\begin{array}{l}\text { Nominal Significance } \\
\text { Level }\end{array}$ & $\begin{array}{l}\text { Sinclair \& Spurr } \\
\text { Approximation }\end{array}$ & $\begin{array}{l}\text { Saddlepoint } \\
\text { Approximation }\end{array}$ \\
\hline 0.283 & 0.95 & 1.3841 & 0.9486 \\
0.346 & 0.90 & 1.1754 & 0.8961 \\
0.399 & 0.85 & 1.0380 & 0.8435 \\
0.774 & 0.50 & 0.5122 & 0.4833 \\
1.249 & 0.25 & 0.2508 & 0.2400 \\
1.933 & 0.10 & 0.1017 & 0.0981 \\
2.492 & 0.05 & 0.0512 & 0.0498 \\
3.079 & 0.025 & 0.0256 & 0.0251 \\
$3.857^{*}$ & 0.01 & $0.010^{* *}$ & 0.0104 \\
& & & \\
\hline
\end{tabular}

Note: $\quad *$ This value was reported as 3.880 by Sinclair and Spurr (1988). The correct value is reported by Anderson and Darling (1954, p. 766) and Lewis (1961, p. 1124).

** This value was reported to only three decimal places by Sinclair and Spurr (1988).

Table 2 provides a detailed comparison of Lewis's (1961) evaluations, and our saddlepoint approximation, for the asymptotic c.d.f. for the Anderson-Darling statistic. We pay special attention to tail-area behaviour, but some illustrative evaluations are also provided in the region of the median. There is extremely close agreement between Lewis's evaluations are the saddlepoint approximation in both tails of the distribution. Consistent with the result for a critical value of 0.774 in Table 1, the saddlepoint approximation is less impressive in the central part of the distribution, though this is of limited concern in terms of the application of the test. 
Table 2. Evaluations of the Asymptotic c.d.f.

\begin{tabular}{lll}
\hline Critical Value & Lewis's Evaluation & Saddlepoint Approximation \\
\hline 0.100 & 0.0000 & 0.0000 \\
0.125 & 0.0003 & 0.0003 \\
0.150 & 0.0014 & 0.0014 \\
0.175 & 0.0042 & 0.0043 \\
0.200 & 0.0096 & 0.0098 \\
0.225 & 0.0180 & 0.0185 \\
0.250 & 0.0296 & 0.0306 \\
0.700 & 0.4412 & 0.4577 \\
0.750 & 0.4815 & 0.4984 \\
0.800 & 0.5190 & 0.5359 \\
0.850 & 0.5537 & 0.5703 \\
3.000 & 0.9726 & 0.9725 \\
3.500 & 0.9846 & 0.9844 \\
4.000 & 0.9913 & 0.9911 \\
4.500 & 0.9950 & 0.9949 \\
5.000 & 0.9971 & 0.9970 \\
5.500 & 0.9983 & 0.9983 \\
\hline
\end{tabular}

\section{CONCLUDING REMARKS}

We have shown that the standard Lugannini-Rice saddlepoint formula provides an accurate approximation to the tail areas of the asymptotic distribution of the Anderson-Darling goodness-of-fit test statistic. The saddlepoint results are superior to the theoretical approximation offered by Lewis (1961). As the limit distribution in question is skewed, as interesting extension of this analysis would be use a non-Normal "base distribution" for the saddlepoint formula. Such a generalization is explored by Wood et al.(1993), and a Chisquare base suggests itself in the present context. 


\section{References}

Anderson, T. W. and D. A. Darling (1952), “Asymptotic Theory of Certain Goodness of Fit Criteria Based on Stochastic Processes", Annals of Mathematical Statistics, 23, 193-212.

D’Agostino, R. B. and M. A. Stephens (eds.) (1986), Goodness of Fit Techniques, Marcel Dekker, New York.

Daniels, H. D. (1954), "Saddlepoint Approximations in Statistics", Annals of Mathematical Statistics, 25, 631-650.

Goutis, C. and G. Casella (1999), "Explaining the Saddlepoint Approximation”, American Statistician, 53, 216-224.

Huzurbazar, S. (1999), "Practical Saddlepoint Approximations", American Statistician, 53, 225-232.

Lahey Computer Systems (1992), Lahey F77L Fortran Programmer's Reference Manual, Lahey Computer Systems, Incline Village, NV.

Lewis, P. A. W. (1961), "Distribution of the Anderson-Darling Statistic”, Annals of Mathematical Statistics, $32,1118-1124$.

Press, W. H., S. A. Teukolsky, W. T. Vetterling, and B. P. Flannery (1992), Numerical Recipes in FORTRAN: The Art of Scientific Computing, $2^{\text {nd }}$. Ed., Cambridge University Press, Cambridge.

Reid, N. (1988), “Saddlepoint Methods and Statistical Inference”(with discussion), Statistical Science, 3, 213-238.

Sinclair, C. D. and B. D. Spurr (1988), "Approximations to the Distribution Function of the AndersonDarling Test Statistic”, Journal of the American Statistical Association, 83, 1190-1191.

Wood, A. T. A., J. G. Booth, and R. W. Butler (1993), "Saddlepoint Approximations to the CDF of Some Statistics With Nonnormal Limit Distributions", Journal of the American Statistical Association, 88, 680-686.

Zolotarev, V. M. (1961), “Concerning a Certain Probability Problem”, Theory of Probability and Its Applications, 6, 201-204. 